\title{
Obesidade: um grande desafio de saúde pública global
}

\author{
Obesity: a major challenge of global public health
}

\begin{abstract}
A s doenças crônicas não transmissíveis (DCNT) são as principais causas de morte no mundo e constituem um problema de saúde pública global. As principais causas das DCNT incluem fatores de risco modificáveis, como tabagismo, consumo nocivo de bebida alcoólica, inatividade física e alimentação inadequada ${ }^{1}$.

As transições demográfica e epidemiológica que caracterizaram as mudanças de perfis de saúde-doença globalmente no século passado, embora em ritmos distintos, foram acompanhadas também de uma transição nutricional, em que as deficiências nutricionais foram "substituídas" pelo excesso de peso. Nesse cenário, emerge um novo problema de saúde pública como é a obesidade.

A obesidade é definida como uma condição de excesso de gordura corporal, e caracterizada como uma doença crônica complexa, multifatorial que envolve fatores ambientais, genéticos, fisiológicos, metabólicos e comportamentais. A prevalência global de sobrepeso e obesidade ${ }^{2}$ aumentou aproximadamente $28 \%$ em adultos e 47\% em crianças no período de 1980 e 2013. O problema não afeta apenas países desenvolvidos, como há agora um aumento significativo em sobrepeso e obesidade em todo o mundo em desenvolvimento ${ }^{3}$. A obesidade tornou-se um grande desafio de saúde global, e a doença hoje é considerada como a epidemia do século XXI . De acordo com dados da Organização Mundial da Saúde, no ano 2014, 1,9 bilhão de adultos estavam acima do peso. Destes, mais de 600 milhões eram obesos. A maioria da população do mundo vive em países onde o sobrepeso e a obesidade causa mais óbitos do que o baixo peso. Além disso, quase 42 milhões de crianças menores de cinco anos tinham sobrepeso em 2010.

No Brasil, conforme dados de 2014 do inquérito VIGITEL (vigilância de fatores de risco e proteção para doenças crônicas por inquérito telefônico), encontrou-se que no conjunto da população adulta das capitais dos estados brasileiros e do Distrito Federal, a frequência de excesso de peso foi de $52,5 \%$, sendo maior entre homens $(56,5 \%)$ do que entre mulheres $(49,1 \%)$. Entretanto, a prevalência de obesidade foi de $17,9 \%$, com índices mais elevados para a população de 35 a 64 anos.

Como resposta ao desafio da obesidade global, é importante continuar investigando o impacto de esta condição nas populações do mundo. Embora alguns países já têm implementado políticas públicas para o sobrepeso e obesidade, se faz necessário que outras nações implementem ações estratégicas para o enfrentamento da obesidade, com o objetivo de promover o desenvolvimento de políticas públicas efetivas para a prevenção e controle deste importante problema de saúde pública.
\end{abstract}

Wilson Cañon Montañez

Doutor. Pesquisador da Faculdade de Medicina da UFRGS, Porto Alegre, Brasil. Professor Associado do Programa de Enfermagem da Universidad de Santander UDES, Bucaramanga, Colômbia.

Rita Mattiello

Doutora. Professora da Faculdade de Medicina da PUCRS, Porto Alegre, Brasil.

\section{REFERÊNCIAS}

1. Schmidt MI, Duncan BB, Azevedo e Silva G, Menezes AM, Monteiro CA, Barreto SM, et al. Chronic non-communicable diseases in Brazil: burden and current challenges. Lancet. 2011;377(9781):1949-61. http://dx.doi.org/10.1016/S0140-6736(11)60135-9

2. Ng M, Fleming T, Robinson M, Thomson B, Graetz N, Margono C, et al. Global, regional, and national prevalence of overweight and obesity in children and adults during 1980-2013: a systematic analysis for the Global Burden of Disease Study 2013. Lancet. 2014; 384(9945): 766-81. http://dx.doi.org/10.1016/S0140-6736(14)60460-8

3. Prentice AM. The emerging epidemic of obesity in developing countries. Int J Epidemiol. 2006; 35(1): 93-9. http://dx.doi.org/10.1093/ ije/dyi272

4. Rössner S. Obesity: the disease of the twenty-first century. Int J Obes. 2002; 26(Suppl): S2-4. http://dx.doi.org/10.1038/sj.ijo.0802209 